\title{
Digital RoF Aided Cooperative Distributed Antennas with FFR in Multicell Multiuser Networks
}

\author{
Xinyi Xu, Rong Zhang, Member, IEEE and Lajos Hanzo, Fellow, IEEE \\ School of ECS., Univ. of Southampton, SO17 1BJ, UK. \\ Tel: +44-23-80-593 125, Fax: +44-23-80-593045 \\ Email: 1h@ecs.soton.ac.uk, http://www-mobile.ecs.soton.ac.uk
}

\begin{abstract}
The achievable throughput of the entire cellular area is investigated, when employing fractional frequency reuse techniques in conjunction with realistically modelled imperfect optical fibre aided distributed antenna systems (DAS). Given a fixed total transmit power, a substantial improvement of the celledge area's throughput can be achieved without reducing the cellcentre's throughput. The cell-edge's throughput supported in the worst-case direction is significantly enhanced by the cooperative linear transmit processing technique advocated. Explicitly, a cell-edge throughput of $\eta=5 \mathrm{bits} / \mathrm{s} / \mathrm{Hz}$ may be maintained for a imperfect optical fibre model, regardless of the specific geographic distribution of the users.
\end{abstract}

\section{INTRODUCTION}

The classic Unity Frequency Reuse (UFR) pattern may be applied by wireless systems in order to maximize the attainable area spectral efficiency at the cost of increasing the co-channel interference level. The Fractional Frequency Reuse (FFR) [1] philosophy is capable of improving the cell-edge Signal-toInterference-plus-Noise-Ratio (SINR) at the cost of a reduced area-spectral-efficiency. As a result, FFR has been adopted in the Third Generation Partnership Project's (3GPP) Long Term Evolution (LTE) initiative [2] and in the Worldwide interoperability for Microwave Access (WiMAX) [3] system.

By contrast, a DAS improves the cell-edge throughput by placing the remote antennas (RA) more close to the cell-edge terminals, hence naturally reducing the pathloss [4]. However, the reduced pathloss will increase the interference imposed on the cell-edge terminals served by the neighbouring RAs. When the MS is roaming near the angle halfway between two adjacent RAs [5], its throughput is reduced, hence we will refer to this as the 'worst-case direction' problem. As a potential remedy, cooperative processing techniques which have been considered for Base Stations (BS) [6] may be adopted for the DAS scenario considered in order to mitigate the InterRA-Interference (IRI). However, the family of BS cooperation techniques still relies on the classic architecture of a single centralized BS covering the entire cell, where the pathloss-and shadow-fading induced problem of the cell-edge users may not be readily solved. Hence, in order to achieve a high throughput for the cell-edge terminals, we propose to combine DASs with cooperative BS processing techniques.

For the practical application of DASs, the Radio over Fibre (RoF) [7] transmission technique may be used for the central BS to RA links. The RoF techniques may be classified into the

Acknowledgements: The financial support of the EPSRC under the auspice of the UK-India Advanced Technology Centre in Wireless Communications as well as that of the EU under the auspices of optimist project and of the UK/China scholarships for excellence programme is gratefully acknowledged.
digital-RoF and analog-RoF families according to the optical modulation depth of the fibre link [8]. In contrast to the analogRoF family, the digital-RoF subclass is capable of avoiding both the inter-modulation distortions of the optical fibre as well as the nonlinearity imposed by the optical components, but this is typically achieved at an increased complexity and cost [9]. Hence in this study we invoke digital-RoF techniques.

Against this background, we quantify the attainable throughput across the entire cellular area in a multicell, multiuser scenario, when employing cooperative techniques beneficially combined both with realistically modelled imperfect optical fibre aided DASs as well as with FFR. More explicity, we introduced the Split-Step Fourier (SSF) method [10] to analyse how the dispersion and nonlinearity of the optical fibre link affect the throughput of the cell-edge area.

Our paper is organised as follows. In Section II, we introduce the system topology and imperfect optical fibre model. In Section III, we outline the received signal model of the noncooperative DAS with FFR system (Section III-A), introduce cooperative linear processing techniques (Section III-B) and present our link level results recorded for a range of practical modulation and coding schemes (Section III-C) followed by our performance evaluations in Section IV. Finally, we conclude our discourse in Section V.

\section{SYSTEM DESCRIPTION}

\section{A. Multicell Multiuser System Topology}

The multicell multiuser scenario based on the FFR scheme considered is illustrated in Fig. 1, which consists of two tiers of nineteen hexagonal cells. We assume symmetry, where every cell has the same system configuration and focus our attention on cell $B_{0}$ of Fig. 1 without any loss of generality. The frequency partitioning strategy of the total available bandwidth $F$ is characterzed by $F_{c} \cap F_{e}=\oslash$, where $F_{c}$ and $F_{e}$ represent the cell-centre's frequency band and the cell-edge's frequency band, respectively. Furthermore, $F_{e}$ is divided into three orthogonal frequency bands $F_{i}, i \in[1,3]$, exclusively used at the cell-edge of one of the three adjacent cells. To simplify the structure, we assume that the number of active MSs $N_{m}$ and RAs $N_{r}$ is the same $\left(N_{m}=N_{r}\right)$, which implies that each RA may support a single MS. The users are assumed to be randomly distributed in the cell-edge area.

Our results not included here for reasons of space economy suggest that the IRI experienced in the local cell dominates the interference experienced in the RoF aided DAS combined with FFR. We also found that the DAS combined with FFR requires a reduced transmit power at each $\mathrm{RA}$, which naturally 


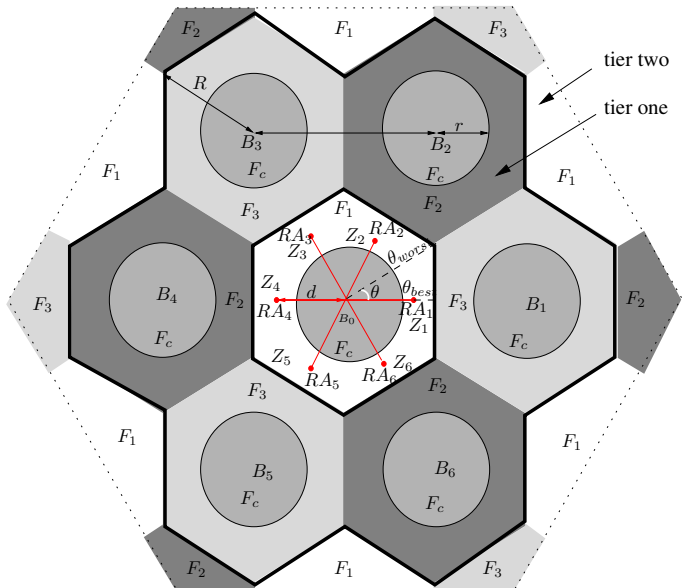

Fig. 1. The toplogy of two tiers of nineteen cells relying on a FFR and DAS arrangement, where $N_{r}=6$ RAs are employed. The structure of the cells in tier two is the same as the ones in tier one.

limits the IRI. Hence, the IRI inflicted by the tier-two cells will be neglected in order to simplify our analysis in the multicell multiuser scenario considered.

\section{B. Imperfect Optical Fibre Model}

We assume that the links from BS $B_{0}$ of Fig. 1 to the RAs are constituted by a realistic imperfect optical fibre. The phase noise imposed by the optical fibre link is supposed to be compensated, hence we only considered the fibre-induced attenuation. Hence, the signal received at the RA $i$ after passing through the optical fibre may be written as: $s_{i}=A_{L} x_{i}+n_{f}$, where $A_{L}, x_{i}$ and $n_{f} \sim \mathcal{C N}\left(0, \sigma_{f}^{2}\right)$ represent the received amplitude of the optical pulse after passing through the optical fibre having a total length of $L$, the transmitted signal and the complex-valued Additive White Gaussian Noise (AWGN), respectively. Furthermore, the so-called pulse-broadening (PB) effect caused by dispersion [10] may additionally imposes an attenuation of the optical signalling pulse, which may affect the attainable throughput of the cell-edge area, when considering our RoF aided DAS combined with FFR system.

The parameter $A_{L}$ takes into account the fibre's dispersion and nonlinearity characterized by the simplified generalized Nonlinear Schrödinger (NLS) equation [10] expressed as:

$$
\frac{\partial A}{\partial z}=\left(-i \frac{\beta_{2}}{2} \frac{\partial^{2}}{\partial t^{2}}+i \xi|A|^{2}\right) A=(\widehat{D}+\widehat{N}) A,
$$

where $A(z, t)$ is the signalling pulse envelope as a function of both the time $t$ and the propagation distance $z$. The term $\widehat{D}$ characterizes the dispersion effect, while $\widehat{N}$ characterizes the nonlinearity effects, as the pulse propagates along the optical fibre. In the term $\widehat{D}, \beta_{2}$ is the group-velocity dispersion (GVD) parameter. In the term $\widehat{N}, \xi$ describes the fibre's nonlinearity.

More explicity, we employ the SSF method [10] for jointly analyzing the attainable throughput of the RoF link. The SSF method delivers an approximate numerical solution, where the dispersive and nonlinear effects observed in Eq (1) may be separated into independent phenomena over a small segmentlength $l$ stretching from $z$ to $(z+l)$ in Eq (1), yielding:

$A(z+l, t) \approx F_{T}^{-1}\left\{\exp [l \widehat{D}(-i \omega)] F_{T}[\exp (l \widehat{N}) A(z, t)]\right\}$, where $F_{T}$ and $F_{T}^{-1}$ denotes the Fourier-transform and inverse Fourier-transform operation, respectively. The Fouriertransform of the dispersion character $\widehat{D}$ is obtained by replacing the operator $\partial / \partial T$ by $-i \omega$ in Eq (1) [10].

\section{RECEIVED SIgNAL OF RoF-DAS-FFR SystemS}

\section{A. RoF aided Non-Cooperative DAS with FFR system}

When non-cooperative DASs are employed, the transmit power $P_{B}$ of the $\mathrm{BS}$ and the transmit power $P_{R}$ of each RA should obey the total transmit power constraint of $P_{B}+$ $N_{r} P_{R}=P$, where $P$ is the full power at the BS of the UFR and FFR scheme.

1) The Cell-Centre Area: The interference at any MS located in the cell-centre area is imposed by the direct wireless links of all BSs $\left\{1, \ldots, N_{b}\right\}$, with $N_{b}$ being the number of BSs. Hence, the received signal may be written as:

$$
y_{c}=\sqrt{P_{B}} \psi_{B 0} h_{B 0} x_{0}+\sum_{j=1}^{N_{b}} \sqrt{P_{B}} \psi_{B j} h_{B j} x_{j}+n,
$$

where $\psi_{B j}, h_{B j}, j \in\left[0, N_{b}\right]$ and $n \sim \mathcal{C N}\left(0, \sigma_{0}^{2}\right)$ represent the combined pathloss plus shadowing based large-scale signal attenuation, small-scale fast Rayleigh fading and the complexvalued AWGN, respectively. More explicitly, we jointly consider both the pathloss and the lognormal shadowing component, which is formulated as: $\psi_{B j}=\left[\rho 10^{\varsigma\left(\sigma_{s}\right) / 10}\right]^{1 / 2}, j \in$ $\left[0, N_{b}\right]$, where $\rho$ denotes the pathloss that obeys a predefined pathloss model [11], while $\varsigma$ denotes a real valued Gaussian random variable having a standard deviation of $\sigma_{s}=8$ $\mathrm{dB}$ [11]. Hence, $G_{B j}=\psi_{B j}\left|h_{B j}\right|$ denotes the equivalent channel gain, while the SINR of any of the MSs near the cell-centre is given by:

$$
\gamma_{c}=\frac{G_{B 0}^{2} P_{B}}{2 \sigma_{0}^{2}+\sum_{j=1}^{N_{b}} G_{B j}^{2} P_{B}} .
$$

2) The Cell-Edge Area: The signal received by MS $i$ at any point in the cell-edge area in the absence of any cooperative techniques, may be written as

$y_{e i}=\sqrt{P_{R}} \psi_{R i} h_{R i} \chi s_{i}+\sum_{k=1, k \neq i}^{N_{r}} \sqrt{P_{R}} \psi_{R k} h_{R k} \chi s_{k}+n$.

Where the power-scaling factor $\chi$ is employed to maintain a constant transmit power at the RAs. Let $A=\chi A_{L}$ and $N_{f}=2 \sigma_{f}^{2} \chi^{2}$ denote the equivalent optical fibre attenuation factor and the equivalent power spectral density of the optical fibre noise, respectively. The details of the parameters $s_{i}$ and $A_{L}$ are introduced above in Section II-B. Then, the SINR at any MS roaming in the cell-edge area is given by:

$$
\gamma_{e i}=\frac{G_{R i}^{2} A^{2} P_{R}}{2 \sigma_{0}^{2}+\sum_{i=1}^{N_{r}} G_{R i}^{2} N_{f} P_{R}+\sum_{k=1, k \neq i}^{N_{r}} G_{R k}^{2} A^{2} P_{R}},
$$

where $G_{R i}=\psi_{R i}\left|h_{R i}\right|$ denotes the equivalent channel gain.

3) Idealistic Received Signal in the Cell-Edge Area: We assume the employment of perfect adaptive beamforming [12], where no IRI is received by the MSs roaming in the cell-edge area. Their reception is only contaminated by the noise $N_{f}$ 
imposed at the optical fibre receiver. Then the upper bound (UB) of the SINR can be rewritten from Eq (6) as:

$$
\gamma_{u}=\frac{G_{R i}^{2} A^{2} P_{R}}{2 \sigma_{0}^{2}+G_{R i}^{2} N_{f} P_{R}} .
$$

4) Benchmarker Systems: Finally, when the classic UFR technique is employed, the received signal model is described by Eq (3), but bearing in mind that the full power $P$ is transmitted by each BS instead of $P_{B}$. When the FFR technique is employed, similarly to the classic UFR, the signal received in the cell-centre area is also described by Eq (3). On the other hand, the signal received in the cell-edge area both from the serving BS and from the cells using the same frequency band in tier-two obeys Eq (3).

\section{B. RoF aided Cooperative DAS with FFR}

To simplify the entire system, only a single omni-directional antenna is applied for each RA. Nonetheless, when jointly designing the Transmit PreProcessing (TPP) matrix T, the $N_{R}$ cooperative RAs operate in a concerted action as a virtual multiple-input and single-output (MISO) or virtual multipleinput and multi-output (MIMO) system. On the other hand, only a single receiver antenna is applied at the MS. We will show that the proposed system architecture is capable of substantially enhancing the throughput achievable in the cell-edge area with our cooperative TPP aided FFR scheme.

Notation: the lower case boldface letters and the upper case boldface letters represent column vectors and matrices, respectively. The superscript $(\cdot)^{T}$ and the $(\cdot)^{H}$ denotes the transposition and the conjugate transpose respectively.

1) Received SINR: There are $N_{m}$ MSs roaming in the celledge area of Fig 1, which are simultaneously supported by the $N_{r}$ RAs $\left(N_{r}=N_{m}\right)$. Provided that the different propagation delays of all the $N_{r}$ RA links measured with respect to all the served $N_{m}$ MSs can be pre-compensated, the vector of received signal can be written as:

$$
\mathbf{y}=\mathbf{H T x}+\mathbf{n},
$$

where $\mathbf{y}_{N_{m} \times 1}$ and $\mathbf{n}_{N_{m} \times 1}$ denote the received signal vector and the circularly symmetric complex Gaussian noise vector. Furthermore, $\mathbf{x}=\left[x_{1}, x_{2}, \cdots, x_{N_{m}}\right]_{1 \times N_{m}}^{T}, x_{i}$ is defined as the signal transmitted from RA $i$ to MS $i$. More explicitly, $x_{i}=$ $\chi_{i} s_{i}, i \in\left[1, N_{r}\right]$ represents the signal passing through the realistic 'lossy' optical fibre, where $\chi_{i}$ and $s_{i}$ is the same as that defined in Section III-A.2. If the central BS has an estimate of the channel matrix $\mathbf{H}=\left[\mathbf{h}_{1}^{T}, \mathbf{h}_{2}^{T}, \cdots, \mathbf{h}_{N_{m}}^{T}\right]_{N_{m} \times N_{r}}^{T}$, we can design a transmit matrix $\mathbf{T}$ for mitigating the IRI, where $\mathbf{T}=\left[\mathbf{t}_{1}, \mathbf{t}_{2}, \cdots, \mathbf{t}_{N_{m}}\right]_{N_{r} \times N_{m}}$ is uniquely and unambiguously determined by $\mathbf{H}$. We also have a dedicated TPP matrix $\mathbf{T}$, which obeys the per RA power constraint of:

$$
\mathbf{T}_{i, \forall} \mathbf{T}_{i, \forall}^{H} \leq \frac{P-P_{B}}{N_{r}},
$$

where $\mathbf{T}_{i, \forall}$ is the row vector of the matrix $\mathbf{T}$.

Furthermore, $\mathbf{h}_{i}=\left[\psi_{1} h_{1}, \psi_{2} h_{2}, \cdots, \psi_{N_{r}} h_{N_{r}}\right]_{1 \times N_{r}}, i \in$ $\left[1, N_{m}\right]$, represents the channel of all the $N_{m}$ RA to MS $i$ links, which takes into account both the large-scale signal attenuation and the small-scale fast Rayleigh fading channel, where $\psi_{i}$ and $h_{i}$ is the same as that defined in Section III-A.2. Hence, a unified discrete-time model for the signal received by MS $i$ may be formulated based on $\mathrm{Eq} \mathrm{(8)} \mathrm{as:}$

$$
y_{i}=\left\|\mathbf{h}_{i} \mathbf{t}_{i}\right\| x_{i}+\sum_{k \neq i}\left\|\mathbf{h}_{i} \mathbf{t}_{k}\right\| x_{k}+n_{i}
$$

When we have $k \neq i, x_{k}$ is the IRI imposed by the transmit signal intended for MS $k$, but received at MS $i$. Hence, the RoF links' SINR encountered at MS $i$ in the cell-edge area may be written as:

$$
\begin{aligned}
& \gamma_{i}=A^{2} \mathbf{h}_{i} \mathbf{t}_{i} \mathbf{t}_{i}^{H} \mathbf{h}_{i}^{H} \times \\
& {[2 \sigma_{0}^{2}+\underbrace{N_{f} \mathbf{h}_{i} \mathbf{t}_{i} \mathbf{t}_{i}^{H} \mathbf{h}_{i}^{H}}_{I R I_{2}}+\underbrace{\left.\left(A^{2}+N_{f}\right) \mathbf{h}_{i}\left(\sum_{k \neq i} \mathbf{t}_{k} \mathbf{t}_{k}^{H}\right) \mathbf{h}_{i}^{H}\right]^{-1}}_{I R I_{1}},}
\end{aligned}
$$

where $A$ and $N_{f}$ represent the equivalent optical fibre attenuation factor and the equivalent power spectral density of the optical fibre' noise, respectively, as defined in Section III-A.2. It can be seen from Eq (11), that for MS $i$, the IRI is composed of two terms. The term $I R I_{1}$ of Eq (11) is the desired signal of MS $k \neq i$ that is also contaminated by the optical fibre link's noise $N_{f}$, which can be mitigated by appropriately designing the corresponding TPP matrix. The other term, namely $I R I_{2}$ of Eq (11), is the optical fibre link's noise contaminating the desired signal of MS $i$, which is the self-inflicted noise, hence the TPP matrix is unable to mitigate it.

2) Linear Transmit PreProcessing: Our design is based on the $\left(N_{m} \times N_{r}\right)$-element channel matrix $\mathbf{H}$ associated with the central BS and the TPP facilitates a low-complexity matchedfilter-based receiver design at each MS. Our linear TPP matrix $\mathbf{T}$ may be written as: $\mathbf{T}=\mathbf{G} \cdot \mathbf{W}$, where $\mathbf{W}_{N_{m} \times N_{m}}$ is a diagonal matrix representing the power [6] allocated for each RA and $\mathbf{G}_{N_{r} \times N_{m}}$ is the linear TPP matrix jointly designed on the basis of $\mathbf{H}$. The TPP matrix $\mathbf{G}$ can be calculated with the aid of different linear preprocessing algorithms. In this paper we consider the minimum mean square error (MMSE) beamformer [6] as our design example.

The classic MMSE beamforming technique strikes an attractive tradeoff between the achievable interference cancellation and noise enhancement, hence it is attractive for practical applications. The TPP weight matrix $\mathbf{G}_{M M S E}=\mathbf{H}^{H}\left(\mathbf{H H}^{H}+\right.$ $\left.\frac{2 \sigma_{0}^{2}}{P_{R}} \mathbf{I}\right)^{-1}$, which is entirely based on the channel matrix $\mathbf{H}$, remains unaffected by the transmit signal. The employment of the TPP matrix $\mathbf{G}_{M M S E}$ mitigates the $I R I_{1}$ component in Eq (11), eliminating the interference inflicted on the desired signal. However, in contrast to the $I R I_{1}$, the link's self-noise imposed by the optical fibre receiver's noise contribution, namely the $I R I_{2}$ terms of Eq (11) cannot be mitigated by the TPP matrix employed. In fact, the self-interference may even be boosted, when the desired signal of MS $i$ is amplified.

\section{System Throughput}

These SINR expressions may be mapped to the ultimate system performance metric formulated in terms of the achievable throughput $\eta$. The throughput may be characterised by the maximum successfully transmitted information rate, which is 
referred to as the system's goodput, given by:

$$
\eta(\gamma)=R_{M} R_{C}\left[1-P_{b l}(b, \gamma)\right]
$$

where $\gamma$ may represent either $\gamma_{c}$ of Eq (4) or $\gamma_{e i}$ of Eq (6) or $\gamma_{u}$ of Eq (7), $R_{M}$ denotes the 'rate' i.e. the throughput of the modulation scheme, while $R_{C}$ is that of the channel code. Still referring to $\mathrm{Eq}(12), P_{b l}(b, \gamma)$ represents the BLock Error Ratio (BLER) corresponding to the particular Modulation and Coding Scheme (MCS) employed. In this paper, we assume that Bit Interleaved Coded Modulation (BICM) [13] is employed, which relies on Gray mapped M-ary Quadrature Amplitude Modulation (QAM) [12], where we have $M=2^{b}$ and $b=2,4,6$ represents the number of bits per QAM symbol. Hence the modem's throughput is $R_{M}=b$. In our paper, we employ $2^{b}$-ary QAM combined with Rate Compatible Punctured Codes (RCPC) [14] having six selected MCSs, namely Mode $[1, \ldots, 6]$ of $\left[R_{M}, R_{C}\right]=$ $[(2,1 / 2),(2,3 / 4),(4,1 / 2),(4,3 / 4),(6,2 / 3),(6,6 / 7)]$. The detailed link-level simulation results is shown in [5].

\section{Performance Evaluation}

\section{A. Simulation Assumptions}

The system topology considered is illustrated in Fig. 1, where each hexagonal cell has a radius of $R$, and the distance between the two adjacent BSs is $D=\sqrt{3} R$, e.g. $\overline{B_{2} B_{3}}=$ $\sqrt{3} R$. We considered the Urban-Macro propagation scenario of [11], where we have $D=3 \mathrm{~km}$ and the pathloss expressed in $\mathrm{dB}$ is $34.5+35 \log _{10}\left(d_{0}\right)$, with $d_{0}$ being the distance between any transmitter and receiver in $\mathrm{km}$. We assume furthermore that the total transmitter power is $P=46 \mathrm{dBm}$ and the noise power at the MS is $-174 \mathrm{dBm} / \mathrm{Hz}$, when an operating in bandwidth of $10 \mathrm{MHz}$ is considered [11]. Moreover, the optical fibre link's normalised Signal-to-Noise-Ratio (SNR) is assumed to be $50 \mathrm{~dB}$ and the length of the optical fibre is assumed to be five times the distance between the BS and RA [15], where we have $L=5 d$, and $d$ is the line-of-sight distance. We opted for $d=0.7 R, \forall i$.

When employing DASs in a FFR-based cellular system, the transmit power of the BS should be sufficiently high to ensure that the average throughput maintained at a distance of $r$ remains exactly as high as that of the classic UFR technique, while the rest of the power is evenly allocated to the RAs. Hence, when considering the $N_{r}=6$ RAs operating in a non-cooperative DAS aided FFR scenario, the resultant power sharing regime of all the transmitters obeys $P_{B}=2 P / 5$, while for the relay we have $P_{R}=P / 10$. By contrast, when considering the $N_{r}=6$ RAs operating in a cooperative DAS assisted FFR scenario, the power constraint of Eq (9) is applied. Finally, the ultimate throughput is obtained by averaging the SINR over 4000 simulation runs, and then substituting it into Eq (12).

\section{B. Cell-edge Area of Non-cooperative DAS with FFR}

The upper and middle figures in Fig. 2 compare the throughput of the classic UFR system, of the classic FFR system and of the non-cooperative RA-aided FFR assisted systems using $N_{r}=6, N_{m}=6$ in both the best and the worst direction.
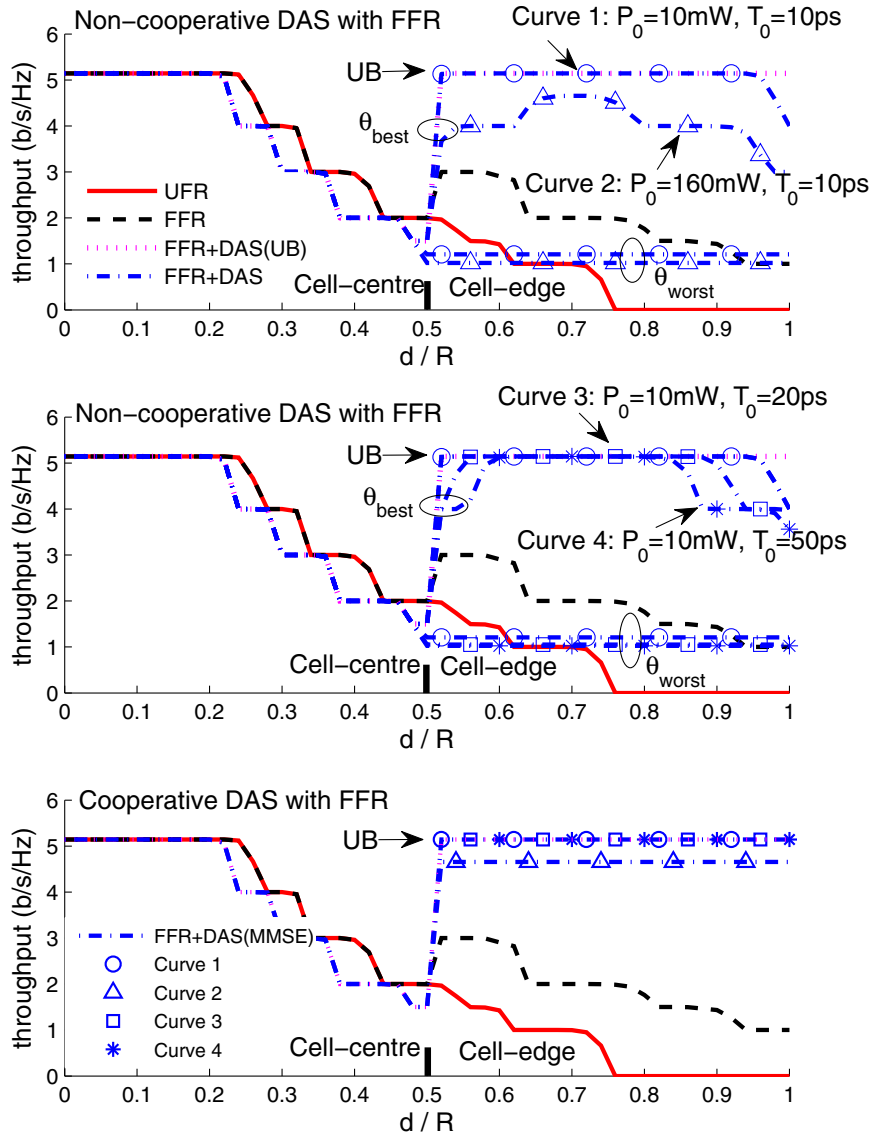

Fig. 2. Throughput comparison of the traditional UFR system, FFR system, the $N_{r}=6$ non-cooperative DAS aided FFR system and of the $N_{r}=6$ cooperative DAS aided FFR system with 4 sets of optical parameters.

1) Best Direction: When the cell-edge area is considered, observe the upper and middle figures in Fig. 2 that the throughput of the conventional UFR scheme becomes lower than $\eta=2$ bits $/ \mathrm{s} / \mathrm{Hz}$, while that of the conventional FFR scheme is in the range of $\eta \in[1,3]$ bits/s/Hz. When the non-cooperative RA-aided FFR-assisted system is employed in conjunction with $N_{r}=6$ RAs, a throughput in the range of $\eta \in[3,5]$ bits/s/Hz is achieved along the best direction. More explicity, when using the parameters of Curves $1-4$ of the upper and middle figures in Fig. 2, suggest that:

Nonlinear Effect: Observe by comparing Curves 1 and 2 of the upper figure in Fig. 2 that assigning a $P_{0}=160 \mathrm{~mW}$ power to the optical pulse makes the nonlinearity-induced impairments dominant, which will broaden the specturm of the optical pulse in the frequency domain. The nonlinearity indirectly results in an increased attenuation for the optical pulses, but this cannot be compensated by an increased transmit power, hence this leads to a boost of the IRI. As a netresult, the system suffers from an approximate throughput loss of $1 \mathrm{bits} / \mathrm{s} / \mathrm{Hz}$ in the cell-edge area along the best direction.

Dispersion Effect: Comparing Curve $3\left(T_{0}=20 \mathrm{ps}\right)$ and 4 ( $\left.T_{0}=50 \mathrm{ps}\right)$ to Curve $1\left(T_{0}=10 \mathrm{ps}\right)$ of the middle figure in Fig. 2, the throughput at the cell-edge remains in the range of $\eta \in[4,5]$ bits $/ \mathrm{s} / \mathrm{Hz}$ is increased from $T_{0}=10 \mathrm{ps}$ to $20 \mathrm{ps}$ and $50 \mathrm{ps}$, the attainable throughput of the cell-edge area is 
reduced. The reason for this observation is that wide pulses are typically broadened to a lesser relative degree along the optical fibre, which also leads to a reduced attenuation both at the optical receiver of the serving RA as well as at the interfering RAs.

2) Worst Direction: When considering the worst direction in both upper and middle figures in Fig. 2, regardless of the specific choice of the four parameter sets, we only achieve a throughput of about $\eta=1 \mathrm{bits} / \mathrm{s} / \mathrm{Hz}$. This is due to the above-mentioned worst-case direction problem [5], which becomes the limiting factor in the case of the conventional non-cooperative RA-aided FFR assisted system.

\section{Cell-edge Area of Cooperative DAS with FFR}

When the cooperative RA-aided FFR-assisted system using the parameters associated with Curves 1, 3 and 4 is considered, observe in the lower figure in Fig. 2 that linear TPP achieves a throughput of $\eta=5 \mathrm{bits} / \mathrm{s} / \mathrm{Hz}$, which is similar to the throughput UB of the RA-aided FFR-assisted system. Hence, when the nonlinearity effects can be ignored, the dispersion does not overly affect the throughput of the wireless channel.

Comparing Curve 2 and Curve 1 in the lower figure in Fig. 2, when using a peak power of $P_{0}=160 \mathrm{~mW}$ for the signalling pulses, the MMSE TPP techniques achieve a slightly higher throughput than $4 \mathrm{bits} / \mathrm{s} / \mathrm{Hz}$. Since the term $I R I_{1}$ in Eq (11) is mitigated by the MMSE TPP, the throughput reduction is imposed by the further attenuation of the received optical signalling pulses inflicted by the fibre's nonlinearity.

\section{Enhancement of Throughput Across the Entire Cell}

In order to observe the attainable throughput improvement $\Delta \eta$ achieved by the cooperative RA aided FFR assisted systems over their non-cooperative counterparts across the entire cell, the resultant throughput contour profile associated with Curve 1 is portrayed in Fig. 3. We configured the cooperative techniques for enhancing the attainable throughput in the celledge area, hence in the cell-centre area we have $\Delta \eta=0$. In the cell-edge area, when considering the cooperative MMSE arrangement for example, there is a significant throughput improvement in the worst-case direction, since we have $\Delta \eta=$ $4 \mathrm{bits} / \mathrm{s} / \mathrm{Hz}$ in the direction $\theta_{\text {worst }}$. When the MS is roaming close to the RA, the throughput improvement achieved by the cooperative MMSE technique remains limited. When however the MS is roaming far from the RA, the benefits of the cooperative techniques become more pronounced.

\section{CONCLUSIONS}

Our work demonstrated that the non-cooperative DAS aided FFR system is capable of gleaning some benefits from the imperfections of the optical fibre, because the dispersion of the optical signalling pulse might indirectly increase the attainable throughput of the cell-edge area. The cooperative RA-aided FFR relying on the linear TPP techniques advocated is capable of efficiently mitigating the IRI in the worst-case direction. As a result, the system may be capable of supporting a throughput of $\eta=5$ bits $/ \mathrm{s} / \mathrm{Hz}$, regardless of the specific geographic userdistribution encountered. Furthermore, the cooperative RAaided FFR system may become tolerant to the dipsersion of the optical pulse, but sensitive to the fibre-induced nonlinearity, if the optical pulse has a high power.

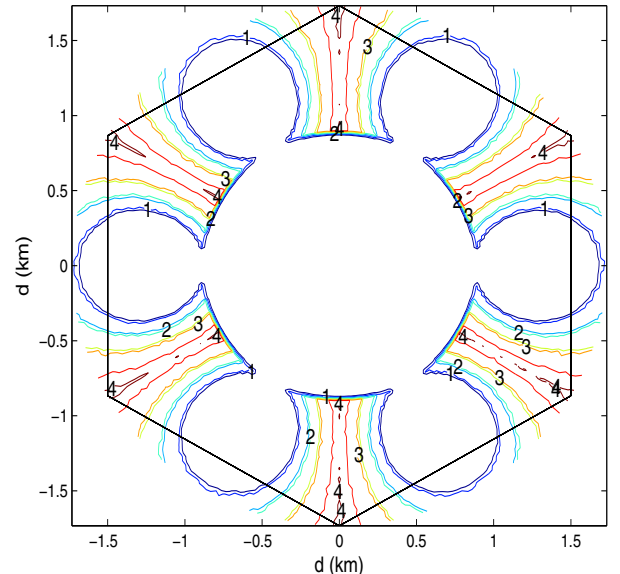

Fig. 3. The enhancement of throughput $\Delta \eta$ contours for the $N_{r}=6$ cooperative DAS aided FFR systems, comparing with non-cooperative DAS aided FFR.

\section{REFERENCES}

[1] Y. Xiang and J. Luo, "Inter-cell interference mitigation through flexible resource reuse in OFDMA based communication networks," Proc. of European Wireless, vol. 43, pp. 1-7, Apr. 2007.

[2] H. Ekstrom, A. Furuskar, J. Karlsson, and et al, "Technical solutions for the 3G long-term evolution," IEEE Communications Magazine, vol. 44, pp. 38- 45, Mar. 2006.

[3] A. Ghosh, D. Wolter, J. Andrews, and R. Chen, "Broadband wireless access with WiMax/802.16: current performance benchmarks and future potential," IEEE Communications Magazine, vol. 43, pp. 129-136, Feb. 2005.

[4] C. Wan and J. Andrews, "Downlink performance and capacity of distributed antenna systems in a multicell environment," IEEE Transactions on Wireless Communications, vol. 6, pp. 69- 73, Jan. 2007.

[5] X. Xu, R. Zhang, and L. Hanzo, "Imperfect radio-over-fibre aided cooperative distributed antennas with fractional frequency reuse," in Proc. of IEEE Vehicular Technology Conference (VTC), Ottawa, Canada, September 2010, pp. 1-5.

[6] H. Zhang and H. Dai, "Cochannel interference mitigation and cooperative processing in downlink multicell multiuser MIMO networks," EURASIP Journal on Wireless Communications and Networking, vol. 2004, pp. 1687-1472, 2004.

[7] D. Wake, M. Webster, G. Wimpenny, and et al, "Radio over fiber for mobile communications," in IEEE International Topical Meeting on Microwave Photonic, Oct. 2004, pp. 157-160.

[8] C. I. Cox, E. Ackerman, G. Betts, and J. Prince, "Limits on the performance of RF-over-fiber links and their impact on device design," IEEE Transactions on Microwave Theory and Techniques, vol. 54, pp. 906 - 920, 2006.

[9] P. A. Gamage, A. Nirmalathas, C. Lim, D. Novak, and R. Waterhouse, "Design and analysis of digitized RF-over-fiber links," Journal of Lightwave Technology, vol. 27, pp. 2052 - 2061, 2009.

[10] G. P. Agrawal, Nonlinear fiber optics, T. Fourth, Ed. Academic Press, 2006.

[11] "Spatial channel model for MIMO simulations," Technical specification group radio access network, 2008, ftp://ftp.3gpp.org/.

[12] L. Hanzo, S. X. Ng, T. Keller, and W. T. Webb, Quadrature Amplitude Modulation: From Basics to Adaptive Trellis-Coded, TurboEqualised and Space-Time Coded OFDM, CDMA and MC-CDMA Systems. Wiley-IEEE Press, 2004.

[13] G. Caire, G. Taricco, and E. Biglieri, "Bit-interleaved coded modulation," IEEE Transactions on Information Theory, vol. 44, pp. 927-946, May 1998.

[14] J. Hagenauer, "Rate-compatible punctured convolutional codes (RCPC codes) and their applications," IEEE Transactions on Communications, vol. 36, pp. 389-400, Apr. 1988.

[15] A. Hekkala, M. Lasanen, I. Harjula, and et al, "Analysis of and compensation for non-ideal RoF links in DAS," IEEE Transactions on Wireless Communications, vol. 17, pp. 52-59, 2010. 